\title{
Propiedades Psicométricas del University Student Engagement Inventory en Estudiantes de Ingeniería Chilenos
}

\author{
Psychometric Properties of the University Student Engagement Inventory in Chilean \\ Engineering Students
}

\author{
Jorge Maluenda Albornoz ${ }^{1}$, Marcela Varas Contreras ${ }^{2}$, Alejandro Díaz Mujica ${ }^{3}$ y Ana B. Bernardo ${ }^{4}$
}

\begin{abstract}
Resumen
El compromiso académico es de interés en educación superior debido a su contribución en el aprendizaje y el logro académico, y su relación inversa respecto de la alienación y el abandono de los estudios. Los objetivos del presente trabajo fueron a) adaptar el University Student Engagement Inventory para su uso en estudiantes de ingeniería chilenos y b) evaluar las propiedades psicométricas del instrumento en esta población. Se realizó un estudio que incluyó 774 estudiantes de ingeniería pertenecientes a 14 áreas disciplinares. Se efectuó una adaptación lingüística y cultural del instrumento al contexto chileno. Se evaluaron modelos de primer y segundo orden, y un modelo bifactorial, además de evaluar la Validez de Criterio. Se observó evidencia que respalda una estructura unidimensional en este instrumento. A partir de estos resultados es posible concluir la utilidad del instrumento en la población de estudio.
\end{abstract}

Palabras clave: compromiso académico, estudiantes universitarios, educación en ingeniería, confiabilidad, validez

\begin{abstract}
Academic Engagement is a variable of interest in higher education due to its contribution to learning and academic achievement, and its inverse relationship with regard to alienation and abandonment of studies. The objectives of the present work were a) to adapt the University Student Engagement Inventory for its use in Chilean engineering students and b) to evaluate the psychometric properties of the instrument in this population. The study included 774 engineering students belonging to 14 disciplinary areas. A linguistic and cultural adaptation of the instrument to the Chilean context was made. First and second order models were evaluated, as well as a bi-factor model, in addition to Criterion Validity. Evidence supporting a onedimensional structure was observed in this instrument. From these results it is possible to conclude the utility of the instrument in the study population.
\end{abstract}

Keywords: study engengement, university students, engineering education, realiability, validity

\footnotetext{
${ }^{1}$ Psicólogo, Magister en Política y Gobierno. Doctorando en Psicología. Doctorado en Psicología, Universidad de Concepción, Chile. Víctor lamas, 129. Concepción, Chile. Tel.: +56982880161. Correo: jorgemaluenda@udec.cl

${ }^{2}$ Ingeniera Civil Informática. Magister en Ciencias de la Computación. Profesora Asistente. Departamento de Ingeniería Civil Informática y Ciencias de la Computación, Universidad de Concepción, Chile. Víctor lamas, 129. Concepción, Chile. Tel.: +56412203566. Correo: mvaras@udec.cl

${ }^{3}$ Psicólogo, Doctor en Psicología. Profesor Titular de la Universidad de Concepción. Víctor lamas 129. Concepción, Chile. Tel.: +569412203955. Correo: adiazm@udec.cl

${ }^{4}$ Psicóloga, Doctora en Psicología. Profesora Titular de Universidad. Departamento de Psicología, Universidad de Oviedo, España. Plaza Feijoo s/n. Tel.: +34985109541. Correo: bernardoana@uniovi.es

Revista Iberoamericana de Diagnóstico y Evaluación - e Avaliação Psicológica. RIDEP · №57 · Vol.4 · 77-90 • 2020

ISSN: 1135-3848 print /2183-6051online
} 


\section{Introducción}

\section{El estudio y medición del compromiso académico}

El estudio del compromiso académico ha cobrado fuerza en educación superior debido a sus efectos positivos sobre el aprendizaje y su relación negativa con aspectos como el bajo logro, la alienación y el abandono de los estudios (Bresó, Schaufeli, \& Salanova, 2011; Medrano, Moretti, \& Ortiz, 2015; Salmela-Aro, Kiuru, Leskinen, \& Nurmi, 2009). Este último, tiene un realce especial debido a que, en Chile, las cifras para el año 2017 dan cuenta de un 21,3\% de abandono a nivel de carrera, $19,3 \%$ a nivel de universidad y $1.8 \%$ a nivel de educación superior (SIES, 2018). El impacto de las consecuencias personales, institucionales y sociales asociadas generan una preocupación importante sobre este tema (Inzunza et al., 2015).

La investigación sobre el compromiso académico en el proceso de enseñanza ha reportado su rol en el favorecimiento del aprendizaje, el desempeño y el logro académico (Gómez, Pérez, Parra, Oritz, et al., 2015; Salanova, Schaufeli, Martínez, \& Bresó, 2009; Schaufeli, Martínez, Marques, Salanova, \& Bakker, 2002). Además, se ha observado que puede operar como un factor protector en el proceso de enseñanza-aprendizaje al relacionarse negativamente con la insatisfacción con los estudios, el burnout y el bajo logro académico (Elmore \& Huebner, 2010; Krause \& Coates, 2008; Wang \& Eccles, 2012). En esta línea, se ha reportado una relación positiva del compromiso académico respecto del desempeño y el aprendizaje, y negativa respecto del abandono de los estudios (Archambault, Janosz, Fallu, \& Pagani, 2009; Chang, Sharkness, Hurtado, \& Newman, 2014; Díaz-mujica et al., 2018; S. Hu \& McCormick, 2012).

Existen dos líneas de trabajo principales en torno al estudio del compromiso académico en educación superior. Una de ellas, proviene del contexto ocupacional, basada en el Modelo de Recursos Bajo Demanda (JD- R) que encuentra sus raíces en la Teoría de la Autodeterminación (Deci, Olafsen, \& Ryan, 2017). Este modelo es transferido al contexto académico por la similitud que algunos autores consideran que existe con el contexto laboral. La actividad estudiantil contemplaría, al igual que la laboral, actividades coercitivas y objetivos específicos que alcanzar (Salanova et al., 2009). La explicación desde el Modelo JD-R sugiere que el compromiso con el trabajo resultaría de la tensión existente entre las demandas laborales y los recursos disponibles por el trabajador para hacer frente a ellas, donde contar con los recursos necesarios para abordar las demandas enfrentadas favorecería el compromiso y motivación con el trabajo, y lo inverso, generaría efectos perniciosos sobre estos factores y la salud (Bakker \& Demerouti, 2014). Este mismo mecanismo operaría en el contexto educativo donde la tensión entre demanda y recursos afecta el nivel de compromiso exhibido por los estudiantes.

Por otro lado, la conceptualización como meta-constructo tridimensional - dimensión conductual, emocional y cognitiva - acuñada por Fredricks, Blumenfeld y Paris, (2003) ha sido desarrollada principalmente en el contexto escolar e intenta integrar distintas líneas de trabajo que consideran dimensiones específicas en el estudio del compromiso académico (Maluenda, Moraga, y Díaz-Mujica, 2019). Su objetivo es integrar las cuatro perspectivas de investigación dominantes: conductual, psicológica, socio-cultural y holística (Maroco, Maroco, Campos, \& Fredricks, 2016).

La perspectiva tridimensional también encuentra sus bases en la Teoría de la Autodeterminación (TAD) que supone que los estudiantes tienen tres necesidades motivacionales fundamentales: autonomía, competencia y relación (Deci et al., 2017). La necesidad de autonomía se satisface cuando el estudiante siente que toma opciones y se motiva por factores intrínsecos más que externos, la necesidad de competencia se favorece cuando la estructura de la clase permite alcanzar los resultados deseados y la necesidad de relación ocurre cuando profesores y pares crean un entorno de apoyo y preocupación (Fredricks \& Mccolskey, 2012).

Por lo tanto, desde esta perspectiva, el compromiso académico es entendido en términos de las acciones, o manifestaciones conductuales, emocionales y cognitivas de la motivación (Fredricks et al., 2003). Estas manifestaciones incluyen específicamente, a) la participación/ involucramiento en las actividades académicas, 
Tabla 1. Procedimientos y resultados obtenidos en la adaptación del USEI

\begin{tabular}{|c|c|c|}
\hline & Procedimiento & Resultados \\
\hline Contenido & $\begin{array}{l}\text { Evaluación realizada por } 20 \text { psicólogos } \\
\text { educacionales a través de escala de } 3 \text { puntos } \\
\text { (Esencial, útil pero no esencial, no necesario). } \\
\text { Cálculo del Content Validity Ratio (CVR). }\end{array}$ & 27 ítems evaluados con $\mathrm{CVR}>0 *$ \\
\hline Criterio & $\begin{array}{l}\text { Validez concurrente UWES-SS, logro académico e } \\
\text { intención de abandono. }\end{array}$ & $\begin{array}{l}\text { UWES-SS }(\mathrm{r}=.99, p<.001) ; \text { Logro }=(\mathrm{B}=1.024 ; \mathrm{SE}=0.125 ; \\
p<.001, \mathrm{OR}=2.79) ; \text { Intención de abandono }=(\mathrm{B}=-0.543 ; \\
\mathrm{SE}=0.102 ; p<.001 ; \mathrm{OR}=0.58)\end{array}$ \\
\hline Constructo & Análisis factorial confirmatorio. & $\begin{array}{l}15 \text { ítems mostraron carga factorial mayor que } .5 \text { en cada } \\
\text { dimensión. El modelo de } 3 \text { dimensiones mostró ajuste } \\
(\chi 2 / \mathrm{df}=2.26 ; \mathrm{CFI}=.97 ; \mathrm{TLI}=.97 ; \mathrm{RMSEA}=.06) .\end{array}$ \\
\hline Confiabilidad & Alfa de Cronbach & $\begin{array}{l}\text { Conductual=.74; Emocional=.88; Cognitivo=.82; } \\
\text { Global }=.88\end{array}$ \\
\hline
\end{tabular}

* CVR>0 implica que la mitad de los jueces consideraron el ítem como esencial.

Elaboración propia basada en el estudio de Maroco et al., (2016).

b) reacciones afectivas positivas y negativas frente a la carrera, estudiantes y docentes, y c) el esfuerzo cognitivo necesario para abordar las tareas complejas y difíciles (Maroco et al., 2016).

Como se señaló previamente, la investigación del compromiso académico ha reportado relaciones con distintas variables importantes para el proceso de enseñanza-aprendizaje, tanto en el plano escolar como universitario. Sin embargo existen investigaciones en que los resultados han sido contrapuestos (Casuso-holgado et al., 2013; Salanova et al., 2009).

Una potencial explicación para este problema tiene relación con la diversidad en las formas en que se ha conceptualizado el compromiso académico que pueden desencadenar en constructos no equivalentes (Fredricks \& Mccolskey, 2012; Maluenda, Moraga, et al., 2019). Asociado a lo anterior, existe diversidad también en las formas de medición, por ejemplo enfocadas en una o más dimensiones, a través de distintos instrumentos y basadas en distintas técnicas (autorreporte, la observación conductual, etc.) (Fredricks et al., 2003).

Un intento por superar estas dificultades ha sido el diseño y validación del SEM - School Engagement Measure de McArthur - (Fredricks, Mccolskey, Manweiler, \& Montrosse-moorhead, 2011) elaborado a partir de la conceptualización como meta-constructo tridimensional, que pretende integrar en el cuestionario los componentes más relevantes del Compromiso Académico en una sola forma de medición. Su versión de 32 ítems, ha sido la versión más utilizada, la cual, ha mostrado buenos resultados en cuanto a validez de constructo, criterio y confiabilidad (Fredericks \& Mccolskey, 2012; Fredericks et al., 2011).
Otra dificultad observada es que los instrumentos diseñados para la medición del Compromiso Académico, en general, son utilizados sin adaptaciones específicas para el contexto universitario (Maluenda \& Pérez, 2018). Por esta razón, es posible que los ítems que incluyen estos instrumentos requieran considerar particularidades de este contexto, además de realizar las adaptaciones lingüísticas necesarias para que se refleje apropiadamente el constructo a evaluar en la población objetivo.

El University Student Engagement Inventory (USEI) es un instrumento construido tomando como base la perspectiva tridimensional de Fredricks et al., (2003) y basado en el SEM. Se compone de 15 ítems con respuesta en escala tipo Likert y valores que fluctúan entre 1 (No me describe bien) y 5 (Me describe muy bien). Estos ítems, mostraron en el USEI una estructura de 3 factores - cognitivo, emocional y conductual acorde a la estructura observada en el SEM. El USEI está disponible en idioma portugués e inglés (Maroco et al., 2016).

Para la elaboración de este instrumento, se realizó un procedimiento que consideró distintas estrategias. Los autores consideraron 15 ítems del inventario original y crearon 17 a partir de los resultados de un focus group realizado con estudiantes universitarios de distintas áreas disciplinares, que tuvo por finalidad recoger: 1) la definición del constructo en el contexto universitario, 2) cómo los estudiantes lo practican a diario en el contexto del trabajo académico, 3) cómo los estudiantes lo practican a diario fuera del contexto de clases. A continuación, sometieron este set de ítems a procedimientos para asegurar su validez de constructo, contenido, 
Tabla 2. Confiabilidad reportada para el UWES-S en estudios con muestra universitaria chilena

\begin{tabular}{lccl}
\hline Muestra & $\begin{array}{c}\text { Involucramiento en } \\
\text { los estudios }\end{array}$ & $\begin{array}{c}\text { Satisfacción con } \\
\text { los estudios }\end{array}$ & Estudio \\
\hline 184 estudiantes de medicina & $\alpha=.83$ & $\alpha=.74$ & Polanco et al., (2014). \\
277 estudiantes de medicina & $\alpha=.85$ & $\alpha=.79$ & Gómez, Pérez, Parra, Oritz, et al., (2015) \\
297 estudiantes de medicina & $\alpha=.91$ & $\alpha=.83$ & Spormann et al., (2015). \\
163 estudiantes de kinesiología & $\alpha=.89$ & $\alpha=.84$ & Hinrichs, Ortiz, \& Pérez, (2016). \\
\hline
\end{tabular}

criterio y evidencia de invariancia métrica (Tabla 1) (Maroco et al., 2016).

El USEI ha sido traducido al español y adaptado para su uso en población universitaria mexicana por Durón-Ramos, M, García-Vásquez, F., Rodríguez, J. y Rodríguez, D. (2018). En el Análisis Factorial Confirmatorio realizado, se observó que el instrumento conserva los 15 ítems de la propuesta de Maroco et al., (2016) manteniendo los tres factores - factor cognitivo, emocional y conductual - y conservando los 5 ítems originales por cada factor. Los índices de bondad del ajuste reportados fueron $\mathrm{BBNF}=.91$, $\mathrm{CFI}=.93, \quad \mathrm{IFI}=.93 \quad$ y $\quad \mathrm{RMSEA}=.062$. Adicionalmente, los autores observaron índices de consistencia interna aceptables para la escala total $(\alpha=.84)$ y para cada factor (cognitivo $\alpha=.71$, emocional $\alpha=.68$ y conductual $\alpha=.77$ ).

Producto de los buenos resultados observados en estas investigaciones respecto del USEI, se observa que éste ofrece una buena prospectiva para evaluar su utilidad en el contexto universitario en otras poblaciones.

\section{Compromiso Académico en Chile}

Una revisión bibliográfica no sistemática de la investigación sobre el Compromiso Académico en el contexto chileno permite observar que la mayor parte de los trabajos realizados en el contexto universitario chileno se han efectuado durante los últimos 5 años, con estudiantes de distintas titulaciones y semestre en curso.

El instrumento que más se ha utilizado ha sido el Utrecht Work Engagement Scale versión estudiante (UWES-S) que cuenta con un cuestionario de 17 ítems y uno de 9 ítems. La versión de 17 ítems ha sido elaborada en castellano por Schaufeli y Bakker, (2003) y es la más utilizada en el contexto universitario chileno. Cuenta con un estudio de evaluación reciente de sus propiedades psicométricas que consideró una muestra de 373 estudiantes de bachillerato y máster en educación de universidades españolas y chilenas (Cachón-zagalaz, Lara-sánchez, Zagalazsánchez, López-Manrique, y González, 2018). Este estudio realizó un Análisis Factorial Exploratorio (AFE) con el uso del método de mínimos cuadrados no ponderados, en el cual, se reporta el uso del test de sedimentación y de una rotación promin. Se observaron 2 factores que explicaron un $58 \%$ de la varianza. A su vez, se reportaron los índices de ajuste del modelo (CFI=.99; GFI=.99; $\mathrm{BIC}=411.900 ; \mathrm{RMSR}=.047$ ).

Por otro lado, el estudio reportó la realización de un Análisis Factorial Confirmatorio sin especificar mayormente el procedimiento. Se reportaron sus índices de ajuste y la reespecificación del modelo $\left(\mathrm{X}^{2}=270.207, p<.01\right.$; RMRSA=.07; CFI=.919; TLI=.902; SRMR=.05). Adicionalmente, en distintas investigaciones previas se ha testeado su confiabilidad mediante el índice Alfa de Cronbach (Tabla 2).

La versión de 9 ítems del UWES-S cuenta con un estudio de propiedades psicométricas realizado por Parra y Pérez, (2010). En este estudio se utilizó AFE considerando como método de extracción el Análisis de Eje Principal, y para la determinación de los factores se usó el criterio de Kaiser-Guttman y el test de sedimentación. A partir de ello, observaron una estructura bifactorial con cargas factoriales que oscilaron entre .69 y .78. Las dimensiones observadas fueron: Predisposición a estudiar y Satisfacción con los estudios. El análisis de confiabilidad se realizó a partir del Coeficiente Alfa de Cronbach encontrando para ambos factores un valor de .87 . Adicionalmente, evaluaron la correlación entre factores encontrando $\mathrm{r}=.69(p<.001)$.

En la línea de trabajo que utiliza el UWES-S se observa la mayor cantidad de estudios, los cuales, establecen la correlación o el valor predictivo del compromiso académico con otras variables importantes para el proceso de enseñanza-aprendizaje.

En estos estudios se ha observado que el compromiso académico muestra correlaciones 
medias con los antecedentes académicos de los estudiantes como las calificaciones de enseñanza media y el puntaje de la PSU (Polanco et al., 2014), la experiencia académica, la percepción académica, la percepción de la atmósfera estudiantil, la percepción de los docentes (Hinrichs, Ortiz, \& Pérez, 2016) y la inteligencia emocional (Ardiles et al., 2019).

Por otro lado, el compromiso académico mostró ser un predictor del promedio ponderado anual de calificaciones (Gómez, Pérez, Parra, Ortiz, et al., 2015), la intención de abandono de los estudios (Díaz-mujica et al., 2018), el aprendizaje autodirigido (Spormann et al., 2015), el rendimiento académico (Oriol-granado, Mendoza-lira, Covarrubias-apablaza, \& Molina-López, 2017) y el ajuste académico (Díaz-mujica et al., 2018).

Por último, con este instrumento, se han observado diferencias estadísticamente significativas en Compromiso Académico en función de la etapa del ciclo vital en que se encuentran los estudiantes, la especialidad que se estudia y el índice de vulnerabilidad del colegio de procedencia (López-Angulo, Maluenda, \& Varas, 2019) y en función del semestre que cursan los estudiantes (Glaría, Carmona, Martín, Pérez, \& Ponce, 2016).

En otra línea de trabajo Aspeé, González y Cavieres-fernández, (2019) desarrollaron un instrumento basado en la National Survey of Student Engagement para estudiantes universitarios que pretendió incluir tres grandes dimensiones: orientaciones académicas, personales-integrales y ciudadanas. El estudio se realizó utilizando una muestra de 108 estudiantes universitarios chilenos siguiendo un procedimiento con distintas etapas. Se realizó un AFE con rotación Varimax que consideró 51 ítems y encontró 15 factores que explicaron el $63.3 \%$ de la varianza. No se especificó el método de extracción de factores. Posteriormente, se eliminaron ítems que no cargaron a ningún factor y se realizó un nuevo AFE, esta vez con rotación promax, obteniendo 11 factores definitivos. La confiabilidad del instrumento se evaluó mediante el índice Alfa de Cronbach obteniendo un valor para la escala total de .80 .

Como es posible observar en los antecedentes presentados la medición del compromiso académico en universitarios chilenos muestra algunas deficiencias metodológicas relevantes de considerar. Entre las más importantes se incluye la utilización de AFE en circunstancias en que existen estudios factoriales previos, la decisión de los factores basados en un solo criterio cuando se recomienda más de uno, el uso de métodos de rotación ortogonal con factores relacionados, muestras pequeñas, la falta de detalle en las decisiones tomadas, y el uso de una puntuación global en una escala sin un modelo que le soporte (Lloretsegura, Ferreres-traver, \& Tomás-marco, 2014).

$\mathrm{Si}$ sumamos a estos problemas las ya mencionadas críticas existentes en el contexto internacional respecto de los instrumentos disponibles, la medición actual del Compromiso Académico, con los instrumentos disponibles, se ha cuestionable. Se profundiza la dificultad relacionada con la no equivalencia de constructos y la dificultad para contrastar los resultados (Fredricks et al., 2003; Maluenda, Moraga, et al., 2019).

Como ya se ha comentado, los resultados iniciales positivos observados en el diseño y validación del USEI con estudiantes universitarios portugueses, y la replicación de estos buenos resultados en una versión en español para estudiantes universitarios mexicanos, motivan la presente investigación para adaptar el USEI al contexto universitario chileno.

Los objetivos de este trabajo son: a) adaptar el USEI para su uso en estudiantes de ingeniería chilenos y b) evaluar las propiedades psicométricas de este instrumento en esta población.

Tabla 3. Distribución de estudiantes por carrera

\begin{tabular}{lc}
\hline Carrera & Porcentaje \\
\hline Ingeniería Civil & 8.39 \\
Ingeniería Electrónica & 6.19 \\
Ingeniería Informática & 7.74 \\
Ingeniería Industrial & 14.45 \\
Ingeniería Aeroespacial & 3.23 \\
Ingeniería en Telecomunicaciones & 7.74 \\
Ingeniería Mecánica & 6.84 \\
Ingeniería de Materiales & 4.52 \\
Ingeniería de Minas & 8.26 \\
Ingeniería Eléctrica & 5.42 \\
Ingeniería Biomédica & 7.61 \\
Plan común & 19.61 \\
\hline
\end{tabular}

\section{Método}

\section{Participantes}

Se obtuvo una muestra por conveniencia compuesta de 729 estudiantes de ingeniería equivalentes al $66,2 \%$ del total de la población 
Tabla 4. Ajustes lingüísticos a ítems originales

\begin{tabular}{ll}
\hline \multicolumn{1}{c}{ Ítem original } & \multicolumn{1}{c}{ Ítem modificado } \\
\hline $\begin{array}{l}\text { 2. Sigo las reglas de la escuela. } \\
\text { 6. No me siento muy realizado en esta escuela. }\end{array}$ & 2. Sigo las reglas de la carrera. \\
$\begin{array}{l}\text { 7. Me siento entusiasmado acerca del trabajo en esta } \\
\text { escuela. }\end{array}$ & 7. Me siento entusiasmado de estudiar esta carrera. \\
8. Me gusta estar en la escuela. & 8. Me gusta estar en esta carrera. \\
$\begin{array}{ll}\text { 9. Me interesa el trabajo en esta escuela. } & \text { 9. Me interesa el trabajo que realizo en esta carrera. } \\
\text { 12. Hablo con personas fuera de la escuela sobre } & \text { 12. Hablo con personas fuera de la carrera sobre } \\
\text { contenidos que aprendo en clases. } & \text { contenidos que aprendí en clases. }\end{array}$ \\
\hline
\end{tabular}

objetivo, con 540 hombres $(74,1 \%)$ y 189 mujeres $(25,9 \%)$. Las edades fluctuaron entre los $17 \mathrm{y}$ los 32 años de edad ( $M=18.66$ años; $D E=5.22$ años). Los estudiantes participantes pertenecen a alguna de 14 posibles carreras de ingeniería y se distribuye como se observa en la Tabla 3.

\section{Diseño y procedimiento}

El estudio se realizó con un diseño de tipo instrumental, realizado en un corte transversal de tiempo (Ato, López, \& Benavente, 2013). La adaptación lingüística y cultural del USEI al contexto chileno se realizó a partir de una traducción al español realizada a partir del instrumento original (Maroco et al., 2016), una evaluación técnica de la traducción y una entrevista cognitiva a estudiantes para evaluar su propia comprensión de los reactivos (SmithCastro \& Molina, 2011).

En primer lugar, se efectuó la traducción del USEI original al idioma español por parte de un psicólogo educacional y un hablante nativo del idioma inglés. Luego, dos psicólogos educacionales evaluaron la traducción efectuada y sugirieron modificaciones lingüísticas.

A continuación, se realizaron entrevistas cognitivas a 10 estudiantes de ingeniería. Esta técnica tuvo el propósito de evaluar el grado de ajuste del USEI para recoger la información en estudiantes universitarios mediante la evaluación de la comprensión del cuestionario y sus reactivos por parte de estos (Smith-Castro \& Molina, 2011). Se realizó el siguiente procedimiento: a) los estudiantes contestaron el instrumento en voz alta, b) indicaron aquellos ítems que representaron alguna complejidad de comprensión, c) propusieron mejoras específicas en su redacción. Los ajustes realizados en función de este procedimiento se observan en la Tabla 4.

La validez de contenido del instrumento con los ajustes antes descritos se evaluó a partir del juicio de jueces especialistas a partir de las respuestas de 4 psicólogos con un promedio de 10 años de experiencia de trabajo en educación superior $(\mathrm{SD}=1.8)$. Para la evaluación de contenido se consideraron los siguientes criterios: a) ítems muy generales, b) redundancia entre ítems, c) afirmaciones poco claras, d) dimensiones no relevantes al constructo, e) ítems poco pertinentes al constructo, f) los ítems no cubren todos los aspectos relevantes del constructo y g) existencia de sesgo de deseabilidad social. Los evaluadores se mostraron en desacuerdo con estos problemas en el instrumento de manera unánime. Además, se les solicitó evaluar el ajuste de los ítems al constructo incluyendo los siguientes criterios éticos: a) Existencia de elementos ofensivos (raza, sexo, nivel económico, etc.) y b) Presencia de estereotipos. En este aspecto, los jueces fueron unánimes en señalar la no presencia de estos problemas.

La validez de constructo se evaluó a partir de un Análisis Factorial Confirmatorio de primer orden, uno de segundo orden y el testeo de un modelo bifactorial.

Para el análisis del modelo de primer orden se utilizó la estructura de tres factores específicos observadas en el estudio original (Maroco et al., 2016) y la adaptación al contexto mexicano (Durón-Ramos et al., 2018).

Teniendo en cuenta que el modelo teórico ha sido formulado a partir de tres factores que componen un factor de mayor jerarquía denominado compromiso académico (Fredricks et al., 2003) y considerando las correlaciones observadas entre los factores específicos reportadas en investigaciones previas (Maroco et al., 2016; Durón-Ramos, et al., 2016) se testeó el modelo de segundo orden y el modelo bifactor, basados en la propuesta teórica de Fredricks et al., 
(2003) que consta de 3 factores específicos y uno general.

En cada caso se analizaron las matrices de correlaciones, cargas factoriales de cada ítem al factor correspondiente, las correlaciones entre factores y los índices de ajuste de cada modelo analizado. Para la extracción de factores se utilizó el método de Mínimos Cuadrados Ponderados con Matriz de Pesaje Diagonal (WLSMV), tal como indica la literatura para el trabajo con variables de tipo categórico (Distefano, Liu, Jiang, \& Shi, 2017; Muthén \& Muthén, 2012). Para los índices de ajuste del modelo se consideró como referencia los valores de corte propuestos para Chi-Cuadrado $\left(\chi^{2}\right)$ no significativo $p>.05$ (Hu et al., 1999), Error de Aproximación Cuadrático Medio (RMSEA) entre .05 y .08 , Índice de Ajuste Comparativo (CFI) e Índice Tucker-Lewis (TLI) mayores de .95 (Hair, Black, Babin, \& Anderson, 2014). Todos estos análisis fueron realizados con el software MPLUS versión 7 (Muthén \& Muthén, 2012).

Adicionalmente, se incorporaron distintos índices estadísticos para valorar la robustez del factor general y evaluar si la contribución de los factores específicos que está por encima del factor general es relevante (Dominguez-Lara \& Rodriguez, 2017).

En la presente investigación se incluyó el análisis del índice Omega Jerárquico tomando como referencia valores $\geq .70$ como evidencia a favor de la unidimensionalidad $\mathrm{y} \geq .30$ a nivel de factor para considerarlas significativas. Se consideraron valores $\geq .70$ en el coeficiente $H$ como indicativos de la confiabilidad de cada factor específico controlando el efecto del factor general. Se consideraron valores $\geq .60$ en el ECV (Explained Common Variance) como indicadores de poca varianza común entre factor general más allá que la del factor general y valores $\geq .80$ en el ECV-I (por ítem) como indicadores de una influencia significativa del factor general sobre los ítems. Además, se incluyó el análisis del PUC (Percentage of Uncontaminated Correlations) como apoyo a la interpretación del ECV considerando valores $\geq .70$ como indicativos de unidimensionalidad. Por último, se incluyeron los coeficientes $\lambda$ para cada factor como medida indicativa de la carga factorial promedio (Dominguez-Lara \& Rodriguez, 2017).
Por otro lado, se incorporó como medida de la validez de criterio la estimación de la correlación entre los puntajes del inventario global y de cada factor, respecto de dos variables criterio: intención de abandono de los estudios y el sentido de pertenencia de los estudiantes a la carrera. La intención de abandono fue recogida a partir de un conjunto de tres preguntas elaboradas por los investigadores (Ej. "Estoy pensando en abandonar la carrera que estoy estudiando"), cuyo estilo de respuesta es de tipo Likert en 7 alternativas (1=Nada; $7=$ Mucho). El sentido de pertenencia de los estudiantes a la carrera de los estudiantes fue medido a partir de un conjunto de tres preguntas elaboradas por los investigadores $(\mathrm{Ej}$. "Me siento perteneciente a esta carrera"), cuyo estilo de respuesta es de tipo Likert en 7 alternativas ( $1=$ Nada; $7=$ Mucho).

\section{Recolección de datos}

El reclutamiento de los estudiantes fue realizado a través de los jefes de carrera para la obtención de permisos y gestionar las aplicaciones, las que fueron realizadas en las salas donde los estudiantes cursan regularmente sus actividades, antes del inicio de una de sus clases, a través de cuestionarios en papel. Previamente se realizó la firma del consentimiento informado que incorporó todos los aspectos éticos necesarios para la investigación en ciencias humanas. No se entregó incentivo por participar. La información fue recolectada durante los meses de marzo y abril del año 2019 (primer semestre académico en Chile).

\section{Resultados}

\section{Testeo de modelos}

El análisis factorial confirmatorio de primer orden se realizó considerando los tres factores que tanto el instrumento original como su adaptación mexicana proponen. El análisis mostró un $\chi^{2}(87)=319.629 ; p<.001$, con un ratio $\chi^{2} / g l=4.03$. El índice RMSEA mostró un valor .057 (95\% IC: .051-.064), el valor del índice CFI fue de .943 y el índice TLI fue de .932 .

Por su parte, el análisis factorial confirmatorio de segundo orden se realizó considerando los mismos tres factores y un factor 


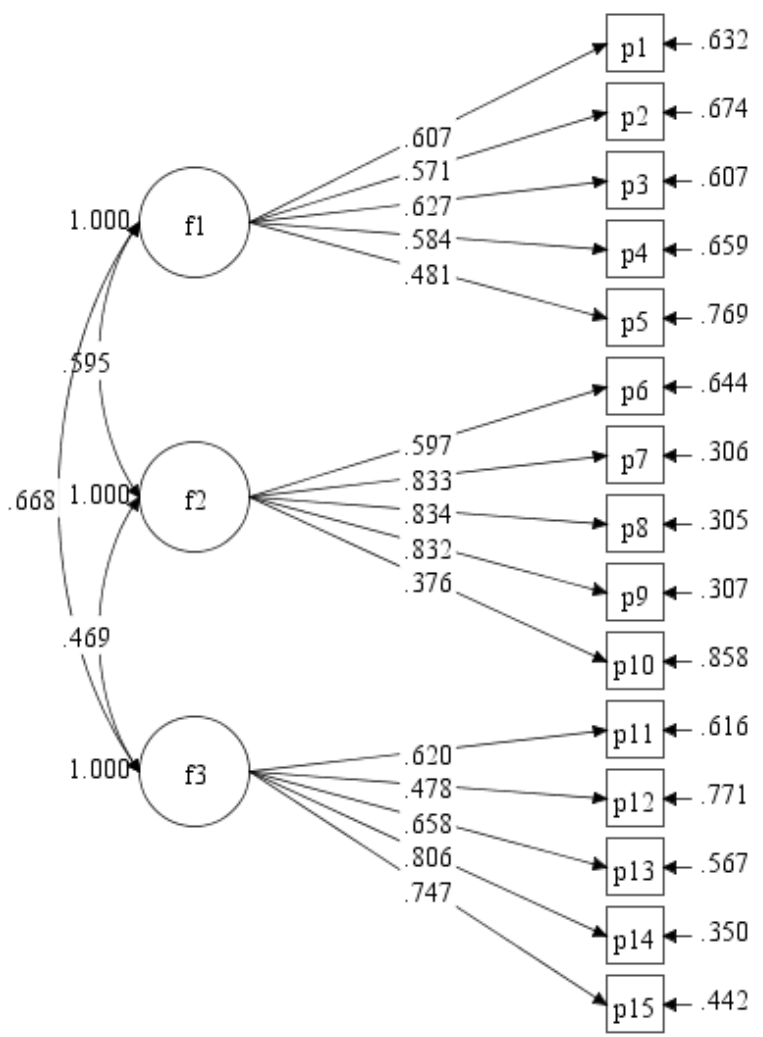

Figura 1. Diagrama Análisis Factorial de Primer Orden con 3 factores

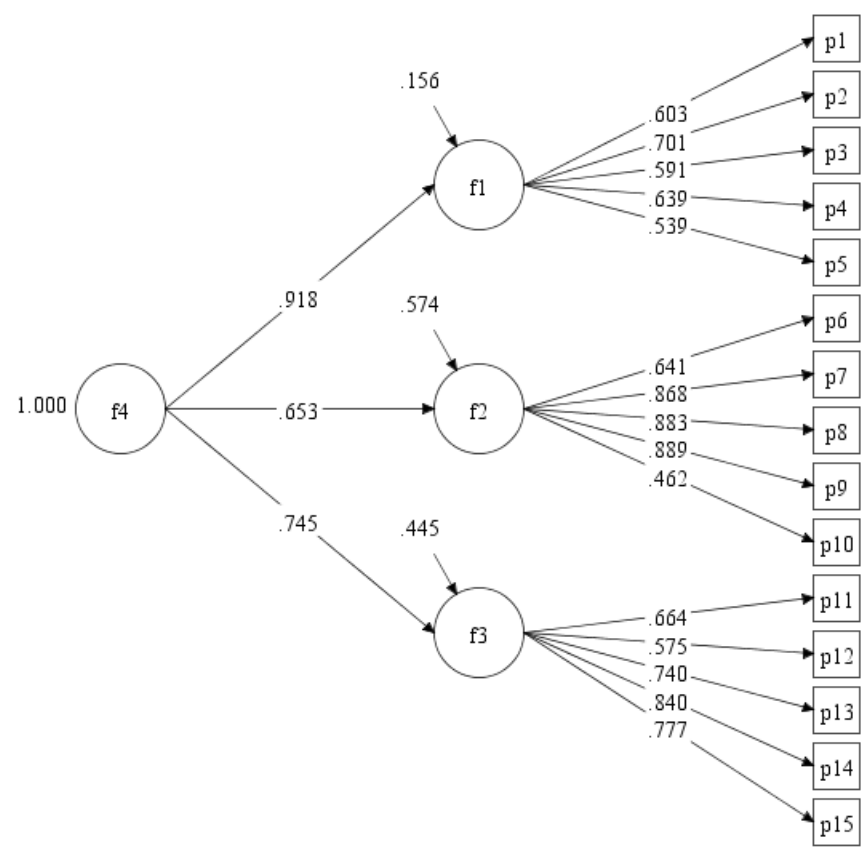

Figura 2. Diagrama Análisis Factorial de Segundo Orden

de segundo orden. El análisis mostró un $\chi^{2}$ $(87)=351.321 ; p<.001$, con un ratio $\chi^{2} / g l=3.67$. El índice RMSEA mostró un valor $.061(95 \%$ IC: .055-.068), el valor del índice CFI fue de .974 y el índice TLI fue de .968. Por último, el análisis bifactorial mostró un $\chi^{2}(75)=210.276$; $p<.001$, con un ratio $\chi^{2} / g l=2.80$. El índice RMSEA mostró un valor .047 (95\% IC: .040-.055), el valor del índice CFI fue de .967 y el índice TLI fue de .954 .

El análisis de los índices de ajuste de la robustez del factor general frente a los factores específicos a partir de $\omega$ y $\mathrm{H}$ mostró índices mayores a los estándares establecidos en todos 


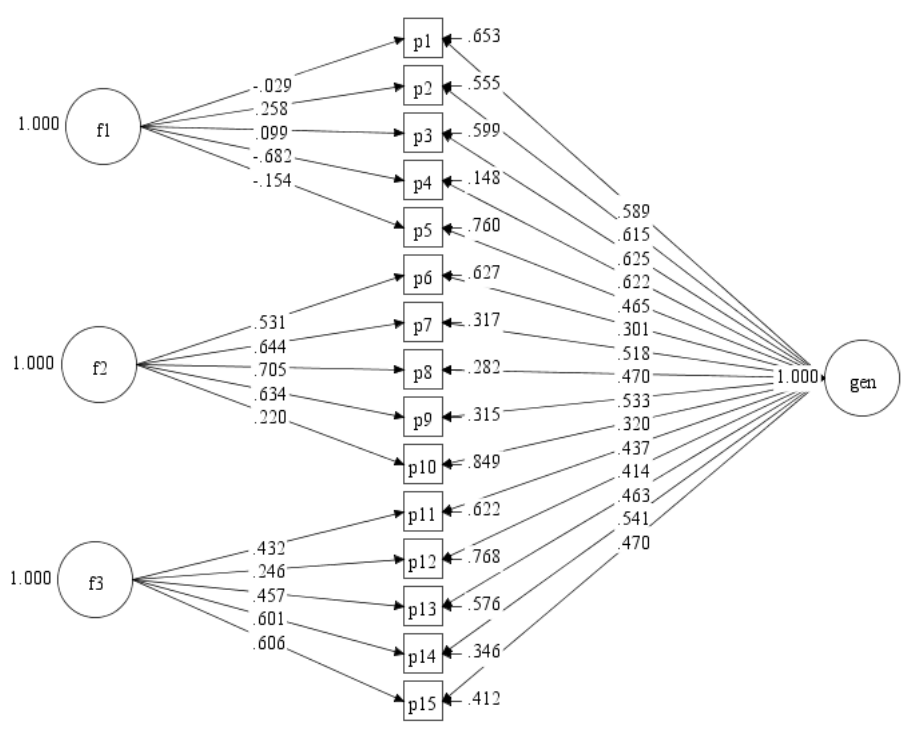

Figura 3. Análisis Bifactorial

Tabla 5. Índices para evaluación de robustez del factor general

\begin{tabular}{lccccc}
\hline & $\omega$ & $\mathrm{H}$ & $\lambda$ & ECV & PUC \\
\hline General & .719 & .843 & .492 & .526 & .714 \\
Factor 1 & .044 & .459 & -.143 & & \\
Factor 2 & .517 & .738 & .547 & & \\
Factor 3 & .403 & .630 & .468 & & \\
\hline
\end{tabular}

Tabla 6. ECV por ítem

\begin{tabular}{lccccc}
\hline Ítems Factor 1 & $\begin{array}{c}\text { ECV-I } \\
\text { Factor 1 }\end{array}$ & Ítems Factor 2 & $\begin{array}{c}\text { ECV-I } \\
\text { Factor 2 }\end{array}$ & $\begin{array}{c}\text { Ítems Factor } \\
3\end{array}$ & $\begin{array}{c}\text { ECV-I } \\
\text { Factor 3 }\end{array}$ \\
\hline 1 & .805 & 6 & .243 & 11 & .506 \\
2 & .850 & 7 & .393 & 12 & .739 \\
3 & .976 & 8 & .308 & 13 & .507 \\
4 & .495 & 9 & .414 & 14 & .448 \\
5 & .901 & 10 & .679 & 15 & .376 \\
\hline
\end{tabular}

los factores excepto el factor 1. El índice ECV bordeó el estándar pero la información que complementa PUC permite indicar que estos valores cumplen el estándar.

En cuanto a los ECV-I se observa que solo los ítems 1, 2, 3 y 5 muestran valores significativos en este criterio.

\section{Validez de criterio}

Al evaluar la correlación de Pearson entre la intención de abandono de la carrera y la escala global se observó una correlación negativa moderada $(r=-.282, p<.001)$. Por su parte la misma variable mostró una correlación negativa y moderada con el factor conductual ( $r=-.173$, $p<.001)$ y una correlación negativa y fuerte respecto del factor emocional $(r=-.49 . p<.001)$. Respecto del factor cognitivo hubo una correlación débil $(r=-.650 ; p<.05)$.
La correlación de Pearson entre el sentido de pertenencia a la carrera y la escala global mostró una correlación positiva moderada $(\mathrm{r}=.43 . p<.001)$. Por su parte la misma variable mostró una correlación positiva moderada respecto del factor conductual $(r=.301$, $p<.001)$, respecto del factor emocional $(r=.417$, $p<.001)$ y del factor cognitivo $(r=.273, p<.001)$.

\section{Discusión y conclusiones}

La presente investigación tuvo como propósitos adaptar el USEI para su uso en estudiantes de ingeniería chilenos y evaluar las propiedades psicométricas de este instrumento en esta población.

La adaptación surgida de la propuesta de los psicólogos especialistas y estudiantes, afectó específicamente a los ítems 2, 6, 7, 8, 9 y 12. Esta 
consistió en la redacción de dichos ítems enfocándolos en el contexto de carrera (titulación) considerando que en Chile, es éste el espacio en que se desenvuelve la experiencia educativa de los universitarios. Este aspecto es distinto del instrumento original donde el contexto de desempeño es la escuela disciplinar en que se dicta la titulación. Los jueces expertos manifestaron que tanto estos cambios como aquellos referidos al contenido y estructura lingüística del instrumento resultante fueron adecuados. Por esta razón, el análisis de la validez de constructo, de criterio y confiabilidad utilizó los ítems modificados.

Al igual que en el estudio original (Maroco et al., 2016), la realización de este proceso incorporando la mirada especialista, experta (examen de jueces) y del estudiante (entrevista cognitiva) fue muy valiosa para ajustar la presentación del USEI al contexto de estudiantes universitarios chilenos. De manera similar al estudio original, el ajuste decantó en una adecuada traducción lingüística y en la verificación de la comprensión de los ítems de parte de los estudiantes.

La evaluación de los tres modelos, uno de primer orden, uno de segundo orden y un modelo bifactor - permitió observar valores para RMSEA aceptables en el modelo de tres factores y adecuados para el modelo bifactor. El modelo de segundo orden mostró valores fuera del estándar establecido. En cuanto a los índices CFI y TLI tanto el modelo de segundo orden como el modelo bifactor mostraron valores acordes al estándar, mientras que el modelo de primer orden mostró valores insuficientes. Los tres modelos mostraron un valor de $\chi^{2}$ significativo, sin embargo, la evaluación del ratio $\chi^{2} / \mathrm{gl}$ mostró un mejor ajuste para el modelo bifactor entre los tres modelos testeados.

El modelo basado en tres factores correlacionados mostró el peor ajuste de los tres modelos testeados. Po su parte, el modelo de segundo orden, formulado siguiendo la estructura teórica propuesta por Fredricks (2003) con un factor conductual, un factor emocional y uno cognitivo interrelacionados, además un factor general (compromiso académico), no mostró el mejor ajuste entre los tres modelos testeados, en contraste con los resultados obtenidos por Maroco et al., (2016). El modelo bifactorial fue el que mostró el mejor ajuste de los tres modelos superando los valores estándar recomendados por la literatura en todos los índices calculados, excepto en $\chi^{2}$. Sin embargo, el ratio $\chi^{2} / \mathrm{gl}$ también fue el mejor en este modelo que en los otros.

Por otro lado, es importante analizar la influencia del factor general y los factores específicos. Tal como señalan Dominguez-Lara \& Rodriguez, (2017), los índices antes reportados solo permiten evaluar el ajuste del modelo, pero no el grado de influencia del factor general, en comparación a los factores específicos, razón por la que se calcularon los índices de ajuste de la robustez del factor general.

$\mathrm{Al}$ respecto, es posible indicar que tanto los índices ECV y PUC a nivel general, como $\omega$ y $\mathrm{H}$ para el factor general y factores específicos, mostraron evidencia a favor de un modelo unidimensional. La excepción se obtuvo en el factor 1, donde para los índices $\omega$ y $\mathrm{H}$ se observaron desajustes.

Los índices ECV-I pueden contribuir a la explicación del comportamiento observado en el factor 1 , en tanto que el ECV-I indica qué porcentaje de la varianza verdadera de cada ítem es explicada por el factor general (DominguezLara \& Rodriguez, 2017). Los resultados mostraron que los ECVI-I para los ítems 1, 2, 3 y 5 mostraron influencia significativa del factor general. Adicionalmente, los valores de $\lambda$ que representan la carga factorial promedio de los factores mostrando un índice general de la influencia de cada factor sobre los ítems (Dominguez-Lara \& Rodriguez, 2017), reflejan que solo en el factor 1 este valor es pequeño.

El conjunto de la evidencia vinculada al desajuste del factor 1 puede estar vinculada a la particularidad en la forma de respuesta de dichos ítems. Este factor está principalmente referido a características propias de la interacción del estudiante con la estructura de la clase. Teniendo en consideración que la recolección de datos fue realizada en los primeros días de abril, fecha en que recién han comenzado las actividades pedagógicas, es posible que la respuesta de los estudiantes se haya basado en la experiencia anterior que han vivido o en hipótesis sobre lo que será su trabajo académico en el semestre, pero no en la experiencia que han tenido. 
En cuanto a la evaluación de la validez de criterio, las correlaciones de la escala total y por factor respecto de las variables criterio incluidas en el estudio muestran los resultados esperados, acorde a los encontrados en investigaciones previas, que reflejan una correlación positiva entre el sentido de pertenencia y el compromiso académico (Maluenda, López, Bernardo, Díaz, \& Moraga, 2019), y una relación negativa entre la intención de abandono y el compromiso académico (Archambault et al., 2009; Chang et al., 2014; Díaz-mujica et al., 2018; S. Hu \& McCormick, 2012). Dada la relación teórica planteada del compromiso académico respecto del sentido de pertenencia y la intención de abandono, estos resultados son indicadores de la capacidad del USEI para representar dicho constructo.

Es importante señalar el contraste en los resultados observados en la evaluación de la validez de criterio utilizada en este estudio (intención de abandono y sentido de pertenencia) y la realizada en el estudio original (Maroco et al., 2016). En ambos estudios se utilizó la intención de abandono como medida observando resultados que apuntan en la misma dirección: una relación inversa entre el Compromiso Académico evaluado con el USEI y la intención de abandono. Sin embargo, en el caso del presente estudio la fuerza de esta relación resultó ser menor. Además, el presente estudio muestra resultados más detallados donde se aprecia que el factor por lejos más correlacionado es el afectivo (correlación alta) frente al factor conductual (correlación moderada) y al factor cognitivo (correlación débil). Este aspecto deja entrever diferencias en la relación de cada factor respecto de la intención de abandono y propone otra prospectiva de investigación que permita identificar qué dimensiones específicas del compromiso académico son mejores predictores de este constructo.

Por otra parte, el presente estudio ofrece una segunda medida de contraste (sentido de pertenencia). Esta medida correlacionó positivamente con el Compromiso Académico evaluado con el USEI de forma similar a resultados previamente observados (Maluenda, López, Varas, et al., 2019). De este modo, se presenta un criterio de correlación positiva y uno de correlación negativa que fortalecen el estudio de la validez de criterio.

\section{Agradecimientos}

El presente trabajo de investigación contó con el financiamiento de Becas Doctorado Nacional CONICYT Folio 21180223. Es parte del proyecto FONDECYT N $\mathrm{N}^{\circ} 1161502$ y fue apoyado por la Facultad de Ingeniería de la Universidad de Concepción.

\section{Referencias}

Archambault, I., Janosz, M., Fallu, J.-S., \& Pagani, L. (2009). Student engagement and its relationship with early high school dropout. Journal of Adolescence, 32, 651-670. https://doi.org/10.1016/j.adolescence.2008.06. 007

Ardiles, R., Alfaro, P., Moya, M., Leyton, C., Rojas, P., \& Videla, J. (2019). La intelifencia emocional como factor amortiguador del burnout académico y potenciador del engagement académico. Revista Electrónica de Investigación En Docencia Universitaria., I(1), 109-128.

Aspeé, J., González, J., \& Cavieres-fernández, E. (2019). Instrumento para medir el compromiso estudiantil integrando el desarrollo ciudadano, una propuesta desde latinoamérica. Revista Complutense de Educación, 30(2), 399-421.

Ato, M., López, J. J., \& Benavente, A. (2013). Un sistema de clasificación de los diseños de investigación en psicología. Anales de Psicología, 29(3 (octubre)), 1038-1059. https://doi.org/10.6018/analesps.29.3.178511

Bakker, A., \& Demerouti, E. (2014). La teoría de las demandas y los recursos laborales. Journal of Work and Organizational Psychology, 29, 107-115. https://doi.org/10.1016/j.rpto.2017.07.003

Bresó, E., Schaufeli, W., \& Salanova, M. (2011). Can a self-efficacy-based intervention increase engagement, and enhance performance? A quasi-experimental study. Higher Education, 61, 339-355. https://doi.org/10.1007/s10734-010-9334-6

Cachón-zagalaz, J., Lara-sánchez, A., Zagalazsánchez, M. L., López-Manrique, I., \& 
González, C. (2018). Propiedades psicométricas de la Utrecht Work Engagement Scale en estudiantes de educación. Suma Psicológica, 25, 113-121.

Casuso-holgado, M. J., Cuesta-vargas, A. I., Moreno-morales, N., Labajos-manzanares, M. T., Barón-lópez, F. J., \& Vega-cuesta, M. (2013). The association between academic engagement and achievement in health sciences students. BMC Medical Education, 13(33), 1-7.

Chang, M., Sharkness, J., Hurtado, S., \& Newman, C. (2014). What matters in College for retaining aspiring scientists and engineers from underrepresented racial groups. Journal of Research in Science Teaching, 51(5), 555580. https://doi.org/10.1002/tea.21146

Deci, E. L., Olafsen, A. H., \& Ryan, R. M. (2017). Self-Determination Theory in Work Organizations: The State of a Science. The Annual Review of Organizational Psychology and Organizational Behavior, 4, 19-43.

Díaz-mujica, A., García, D., López, Y., Maluenda, J., Hernández, H., \& Pérez-Villalobos, M. (2018). Mediación del ajuste académico entre variables cognitivo-motivacionales y la intención de abandono en primer año de universidad. In Octava conferencia latinoamericana sobre el abandono en la educación superior. (pp. 213-222). Panamá City.

Dominguez-Lara, S., \& Rodriguez, A. (2017). Interacciones. Interacciones Revista de Avances En Psicología, 3(2), 59-65. https://doi.org/10.24016/2017.v3n2.51

Elmore, G., \& Huebner, E. (2010). Adolescent's satisfaction with school experiences: Relationships with demographics, attachment relationships, and school engagement behavior. Psychology in the Schools, 47(6), 525-537. https://doi.org/10.1002/pits

Fredricks, J., Blumenfeld, P., \& Paris, A. (2004). School engagement: Potential of the concept, state of the evidence. Review of Educational Research, 74(1), 59-109.

Fredricks, J., \& Mccolskey, W. (2012). The measurement of student engagement: A comparative analysis of various methods and student self-report instruments. In C. Christenson, S, Reschly, A., Wylie (Ed.),
Handbook of Research on Student Engagement. (pp. 763-782). New York: Springer-Verlag. https://doi.org/10.1007/9781-4614-2018-7

Fredricks, J., Mccolskey, W., Manweiler, J., \& Montrosse-moorhead, B. (2011). Measuring student engagement in upper elementary through high school: A description of 21 Instruments. Issues \& Answers, (98), 1-89.

Glaría, R., Carmona, L., Martín, S., Pérez, C., \& Ponce, P. (2016). Burnout y engagement académico en fonoaudiología. Investigación En Educación Médica, 5(17), 17-23. https://doi.org/10.1016/j.riem.2015.08.006

Gómez, P., Pérez, C., Parra, P., Oritz, L., Matus, O., McColl, P., ... Meyer, A. (2015). Relación entre el bienestar y el rendimiento académico en alumnos de primer año de medicina. Revista de Médica de Chile, 143, 930-937.

Gómez, P., Pérez, C., Parra, P., Ortiz, L., Matus, O., McColl, P., ... Meyer, A. (2015). Relación entre el bienestar y el rendimiento académico en alumnos de primer año de medicina. Revista Médica de Chile, (143), 930-937.

Hair, J., Black, W., Babin, B., \& Anderson, R. (2014). Multivariate data analysis (Seventh Ed). Edinburg Gate: Pearson.

Hinrichs, C., Ortiz, L., \& Pérez, C. (2016). Relación entre el bienestar académico de Estudiantes de Kinesiología de una universidad tradicional de Chile y su percepción del ambiente educacional. Formación Universitaria, 9(1), 109-116. https://doi.org/10.4067/S071850062016000100012

Hu, L., Bentler, P. M., \& Hu, L. (1999). Cutoff criteria for fit indexes in covariance structure analysis: Conventional criteria versus new alternatives. Structural Equation Modeling, 6(1), 1-55.

https://doi.org/10.1080/10705519909540118

Hu, S., \& McCormick, A. C. (2012). An engagement-based student typology and its relationship to college outcomes. Research in Higher Education, 53, 738-754. https://doi.org/10.1007/s11162-012-9254-7

Inzunza, B., Ortiz, L., Pérez, C., Torres, G., Meyer, P., Matus, O., ... Durán, C. (2015). Estructura factorial y confiabilidad del Cuestionario de Satisfacción Académica en 
Estudiantes de Medicina Chilenos. Revista Iberoamericana de Diagnóstico y Evaluación - e Avaliação Psicológica, 2(40), 73-82.

Krause, K. L., \& Coates, H. (2008). Students' engagement in first-year university. Assessment and Evaluation in Higher Education, 33(5), 493-505. https://doi.org/10.1080/02602930701698892

Lloret-segura, S., Ferreres-traver, A., \& Tomásmarco, A. H. I. (2014). El análisis factorial exploratorio de los ítems: Una guía práctica, revisada y actualizada. Anales de Psicología, 30(3), 1151-1169.

https://doi.org/10.1177/001452469000100407

López-Angulo, Y., Maluenda, J., \& Varas, M. (2019). Factores personales e institucionales vinculados con el compromiso académico en estudiantes de ingeniería. IX Congreso Lationamericano Sobre Abandono En Educación Superior.

Maluenda, J., López, Y., Bernardo, A., Díaz, A., \& Moraga, F. (2019). Predictores psicosociales de la intención de abandono en estudiantes de ingeniería chilenos. In $I X$ Congreso Lationamericano sobre Abandono en Educación Superior. Bogotá.

Maluenda, J., López, Y., Varas, M., Bernardo, A., Díaz, A., \& Moraga, F. (2019). Predictores psicosociales de la intención de abandono en estudiantes de ingeniería chilenos. In $I X$ Congreso Lationamericano sobre Abandono en Educación Superior. Bogotá.

Maluenda, J., Moraga, F., \& Díaz-Mujica, A. (2019). El rol del estudiante en el fenómeno del compromiso académico en educación superior. Wimblu, 14(1), 81-94.

Maluenda, J., \& Pérez, M. V. (2018). Comportamientos asociados al compromiso académico desde la experiencia de estudiantes de ingeniería. In Octava Conferencia Latinoamericana sobre Abandono en la Educación Superior. Panamá City.

Maroco, J., Maroco, A. L., Campos, J. A. D. B., \& Fredricks, J. A. (2016). University student's engagement: Development of the University Student Engagement Inventory (USEI). Psicologia: Reflexão e Crítica, 29(1), 21. https://doi.org/10.1186/s41155-016-0042-8

Medrano, L., Moretti, L., \& Ortiz, A. (2015). Medición del engagement académico en estudiantes universitarios. Revista Iberoamericana de Diganóstico y Evaluación - e Avaliação Psicológica., 2(40), 114-124.

Oriol-granado, X., Mendoza-lira, M., Covarrubias-apablaza, C., \& Molina-López, V.-M. (2017). Emociones positivas, apoyo a la autonomía y rendimiento de estudiantes universitarios: El papel mediador del compromiso académico y la autoeficacia. Revista de Psicodidáctica, 22(1), 45-53. https://doi.org/10.1016/S11361034(17)30043-6

Parra, P., \& Pérez, C. (2010). Propiedades psicométricas de la escala de compromiso académico , UWES-S (versión abreviada), en estudiantes de psicología. Revista de Educación En Ciencias de La Salud, 7(2), 128-133.

Polanco, A., Ortiz, L., Pérez, C., Parra, P., Fasce, E., Matus, O., \& Torres, G. (2014). Relación de antecedentes académicos y expectativas iniciales con el bienestar académico de alumnos de primer año de medicina, 17(4), 205-211.

Salanova, M., Schaufeli, W., Martínez, I., \& Bresó, E. (2009). How obstacles and facilitators predict academic performance: The mediating role of study burnout and engagement. Anxiety, Stress \& Coping, 1, 118.

https://doi.org/10.1080/10615800802609965

Salmela-Aro, K., Kiuru, N., Leskinen, E., \& Nurmi, J. E. (2009). School burnout inventory (SBI) reliability and validity. European Journal of Psychological Assessment, 25(1), 48-57.

https://doi.org/10.1027/1015-5759.25.1.48

Schaufeli, W., Martínez, I., Marques, A., Salanova, M., \& Bakker, A. (2002). Burnout and engagement in university studentes. A cross-national study. Journal of CrossCultural Psychology, 33(5), 464-481.

SIES. (2016). Informe retención de ler año pregrado cohortes 2011-2015. Santiago, Chile. Retrieved from https://biblioteca.digital.gob.cl/bitstream/hand le/123456789/693/informe retencion_sies_2016.pdf?sequence=1\&isAllo wed $=\mathrm{y}$

SIES. (2018). Informe retención de ler año de 
pregrado. Santiago.

Smith-Castro, V., \& Molina, M. (2011). Cuaderno metodológico 5: La entrevista cognitiva: Guía para su aplicación en la evaluación y mejoramiento de instrumentos de papel y lápiz. San José, Costa Rica.: Instituto de Investigaciones Psicológicas, Universidad de Costa Rica. ISSN.

Spormann, C., Pérez, C., Fasce, E., Ortega, J., Bastías, N., Bustamante, C., \& Ibáñez, P. (2015). Predictores afectivos y académicos del aprendizaje autodirigido en estudiantes de medicina. Revista Médica de Chile, 143, 374382.

Wang, M. Te, \& Eccles, J. S. (2012). Social support matters: Longitudinal effects of social support on three dimensions of school engagement from middle to high school. Child Development, 83(3), 877-895. https://doi.org/10.1111/j.1467-

8624.2012.01745.x 